to high-power observations, with which the image is somewhat blurred, and if the objective is of particularly short focal length the thickness of the polaroid becomes an obstacle. For the average specimen, however, when one is searching for doubly refractile material, the 23 in. objective is the power of choice, and with this there is no difficulty.

To protect the polaroid analyser from minor surface damage and to facilitate its manipulation, an ordinary aluminium screw cap with a single central aperture (as used for blood culture bottles) is taken and the rubber seal replaced with a disc of polaroid. A hole of 6 to $7 \mathrm{~mm}$. diameter is fully adequate and needs no enlarging. For use, the cap is placed inverted on the slide.

A practical advantage of this is that when the objective is focused it dips inside the rim of the cap, and, by so retaining it in position, one has complete freedom of movement for the mechanical stage, and the rotation of the analyser takes place on a central axis. The polaroid can be conveniently held in position in the cap by cutting an appropriate aperture in the original rubber seal and replacing that. A light source of relatively high intensity is, of course, to be preferred.

\section{A Digestion Rack for Kjeldahl Flasks}

\author{
R. F. A. DEAN
}

From the Medical Research Council Group for Research itr Infantile Malnutrition, Mulago Hospital, Kampala, Uganda

(RECEIVED FOR PUBLICATION AUGUST 31, 1956)

A simple and efficient rack for heating Kjeldahl flasks is often needed in many laboratories. Racks heated electrically, and in which a separate control is fitted for each flask, are often advocated, but are very expensive and in practice seldom give good service for a long time. Heating by gas has the advantage that great variations in temperature are possible and control very easy.

The rack to be described has been in use in the laboratory of the Group for the last year, and has proved satisfactory. It can be made with modest workshop facilities, and the cost for materials is trifling. The model described takes eight flasks of $50 \mathrm{ml}$. capacity, which are heated on a gas burner of the ring type. It is illustrated in the photograph (Fig. 1).

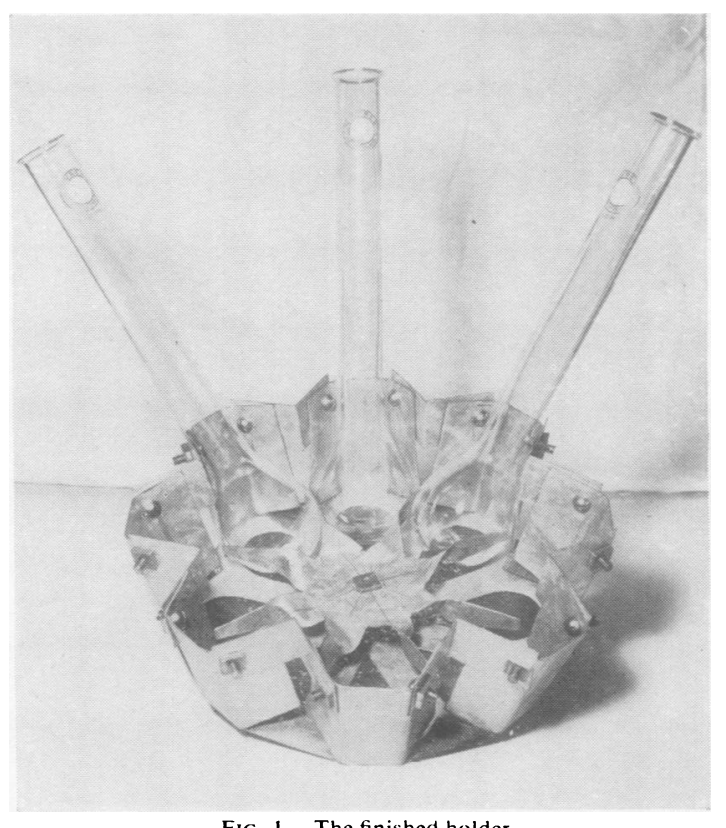

Fig. 1.-The finished holder.

\section{Construction}

All parts are cut from galvanized iron sheet, $1 \mathrm{~mm}$. (1/32 in.) thick. They are : (a) An octagonal base with wings at each corner that form recesses to hold the flasks; 
(b) strips to keep the wings in place; and (c) a starshaped separator.

A square is cut of $30.5 \mathrm{~cm}$. (12 in.) side. Its diagonals, and lines joining the mid-points of opposite sides, are drawn. A template traced from Fig. 2 is then laid, with

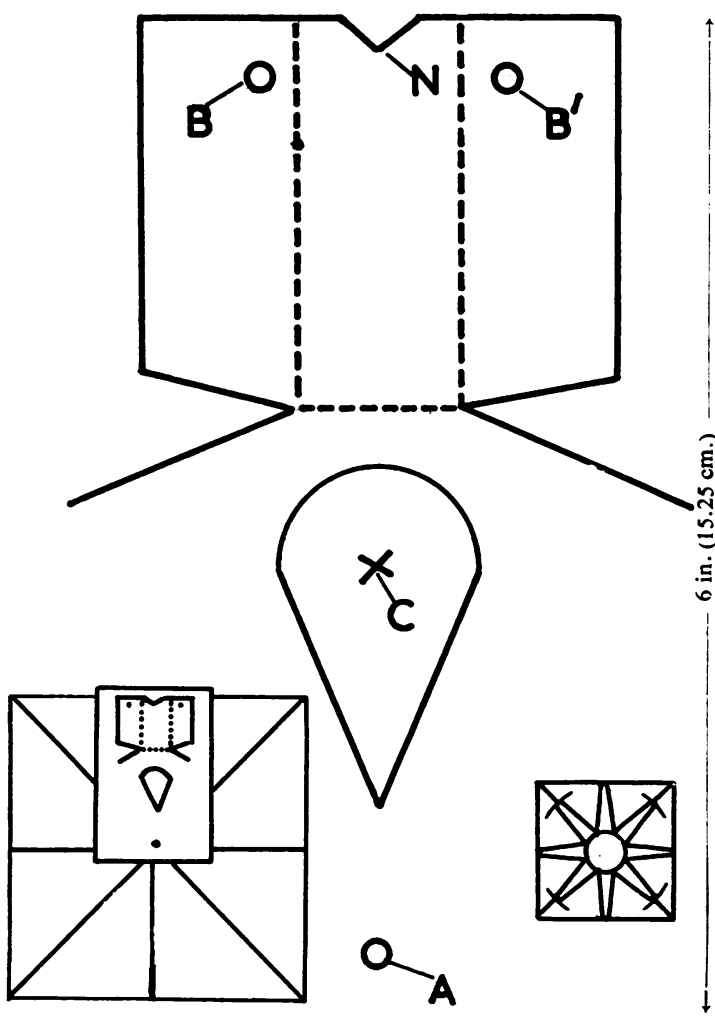

Fig. 2.-Template for the octagonal base. A, B and $B^{\prime}$ are holes for bolts. The niche $\mathbf{N}$ is optional. It facilitates the use of the template and lowers slightly the neck of the flask. The model illustrated (Fig. 1) was made without it. The inset left shows the template in position for making out the first wing, and the inset right shows the lay-out of the star-shaped separator. the point $\mathrm{A}$ at the intersection of the lines (the centre of the sheet), symmetrically across each of the lines in turn so that eight exactly equal sections are marked out. The dotted and solid lines on the template are marked on the sheet. The dotted lines indicate bends and the solid lines indicate where it is necessary to cut with tin shears. Holes are drilled at the points $A, B$, and $B^{\prime}$ to take machine bolts. Holes are made in the base with a hole saw $34 \mathrm{~mm}$. (1 in.) in diameter centred on the point $\mathrm{C}$, and the openings are finished with the shears or a cold chisel.

The eight wings are bent upwards and their sides are bent in. The wings are held together by strips $7 \mathrm{~cm}$. long by $2.5 \mathrm{~cm}$. wide, bent at right angles half-way along, and drilled $0.5 \mathrm{~cm}$. from each end. The strips are bolted to the wings, and the bends in the strips should then be 63 $\mathrm{mm}$. (1-27/32 in.) apart.

An eight-armed star is cut from a square of side $14 \mathrm{~cm}$. ( $5 \frac{1}{2}$ in.) with arms $5 \mathrm{~cm}$. (2 in.) long, $1 \mathrm{~cm}$. across at their free ends, and $2 \mathrm{~cm}$. across at their bases. A hole for a bolt is made in the middle of the body of the star, and each arm is twisted with pliers so that it is at right angles to the body. The arms form the partitions between the flasks. The star is held $2.5 \mathrm{~cm}$. above the base by a bolt $4 \mathrm{~cm}$. long, and adjusted so that the arms lie exactly between the corners of the wings. The corners should be bent slightly outwards.

The holder is turned upside down and a piece of finemesh gauze $15.25 \mathrm{~cm}$. (6 in.) square is laid on the base. It is held in place by turning over the corners where they project beyond the sides of the base. The holder is turned right side up, and a Kjeldahl flask is pressed firmly into each of the holes in turn, to make a slight depression into which the flask fits snugly.

Handles could obviously be fitted to opposite wings if desired, but have not in practice been found necessary. After the racks have been in continuous use for several months, the gauze may have to be renewed, but no other change has had to be made. The angle at which the flasks are held seems to reduce " bumping " and splashing to a minimum, but it is an advantage to add a single glass bead to the contents of each flask. Small glass funnels inserted in the mouths of the flasks provide a simple means for increasing the condensing capacity. 